\title{
Transient Solubility Gradients Mediate Oversaturation during Solvent Exchange
}

\author{
Beng Hau Tan $\odot,{ }^{1}$ Claus-Dieter Ohl $\odot,{ }^{2, *}$ and Hongjie An $\oplus^{3, \dagger}$ \\ ${ }^{1}$ KB Corporation, 7500A Beach Road, 199591 Singapore \\ ${ }^{2}$ Otto von Guericke University Magdeburg, Institute of Experimental Physics, Universittsplatz, 2, 39016 Magdeburg, Germany \\ ${ }^{3}$ Queensland Micro and Nanotechnology Centre, Griffith University, 170 Kessels Road, Nathan, Queensland 4111, Australia
}

(Received 25 August 2020; accepted 28 April 2021)

\begin{abstract}
Solvent exchange facilitates high-density nucleation of sessile nanodroplets or nanobubbles by successively wetting a surface with two solvents of contrasting solubility with respect to a target species. Yet the key physical mechanisms underlying its efficacy have yet to be theoretically explained. We develop a minimal model for solvent exchange, for the prototypical example of water and ethanol as the solvents and nitrogen as the target species. Our calculations show that solvent exchange is mediated by transient solubility gradients that dominate over the intrinsic concentration gradient of nitrogen in the incipient moments after exchange. Solubility gradients advect nitrogen toward the substrate during ethanol-water exchange but away from it in water-ethanol exchange, consistent with the directionality observed in experiments.
\end{abstract}

DOI: 10.1103/PhysRevLett.126.234502

The production of mesoscopic volumes of sessile bubbles and droplets $[1,2]$ is important for the development of engineering technologies such as drag reduction or colloidal manufacturing [3-5]. Among known techniques to produce nanobubbles or nanodroplets [6-9], solvent exchange is distinguished by its simplicity, repeatability, and efficacy. It triggers abundant and high-coverage nucleation on surfaces simply by successively wetting two liquids of contrasting solubility (with respect to a target chemical species) under confined flow, eschewing specialized nanoscale equipment and protocols.

Despite sustained research interest [10-19] in the 20 years since its serendipitous discovery by Lou et al. [20], solvent exchange remains poorly understood. Experiments show that solvent exchange is directional, producing nucleation only when a good solvent is displaced by a poor one; reintroducing the good solvent dissolves the nucleated objects $[10,21-26]$. Yet it is paradoxical that good-poor exchange triggers nucleation through promoting oversaturation. In the canonical case of nanobubble formation during ethanol-water exchange [see Fig. 1(a)] the putative concentration gradient of nitrogen is directed from ethanol to water, which should in principle evacuate dissolved nitrogen away from the substrate, instead of accumulating adjacent to it. In this Letter we present a minimal but perspicuous model for solvent exchange that resolves the salient physical mechanisms of solvent exchange and correctly captures its directionality.

The key parameter that determines the efficacy of solvent exchange is the oversaturation,

$$
\zeta=c / c_{s}-1
$$

of the target species in a given liquid, where $c$ is the dissolved concentration of the species in the liquid and $c_{s}$ is its saturation concentration or solubility. Physically, oversaturation controls the free energy barrier $\Delta G$ for the target species' nucleation (as a bubble or droplet); thermodynamic calculations \{see Ref. [28] and Fig. S1 of the Supplemental Material [29]\} show that $\Delta G \sim 1 / \zeta^{2}$. However, $\zeta$ is nontrivial to measure experimentally, even for atmospheric gases, because commercial dissolved gas concentration devices detect oxygen (not nitrogen, the dominant component in atmospheric gas) and function only in pure water.

The best known estimate of oversaturation generated in solvent exchange is that of An et al. [27], for the case of dissolved nitrogen in water-ethanol mixtures during the nucleation of surface nanobubbles. Defining $x_{e}$ as the mole fraction of ethanol in water, their approach determines $c\left(x_{e}\right)$ by proportionally weighting the saturation concentrations of
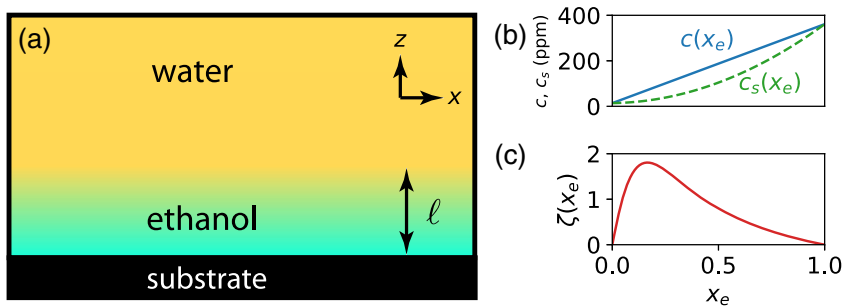

FIG. 1. (a) Schematic of the solvent exchange problem-here a good solvent of nitrogen, ethanol, is replaced by a poor solvent, water. (b),(c) The An model of oversaturation generation [27] for water-ethanol mixing compares $c\left(x_{e}\right)$ and $c_{s}\left(x_{e}\right)$ [Eq. (2)] for all mole fractions $0<x_{e}<1$ of ethanol in water, yielding [via Eq. (1)] a maximum of $\zeta \approx 1.8$. 

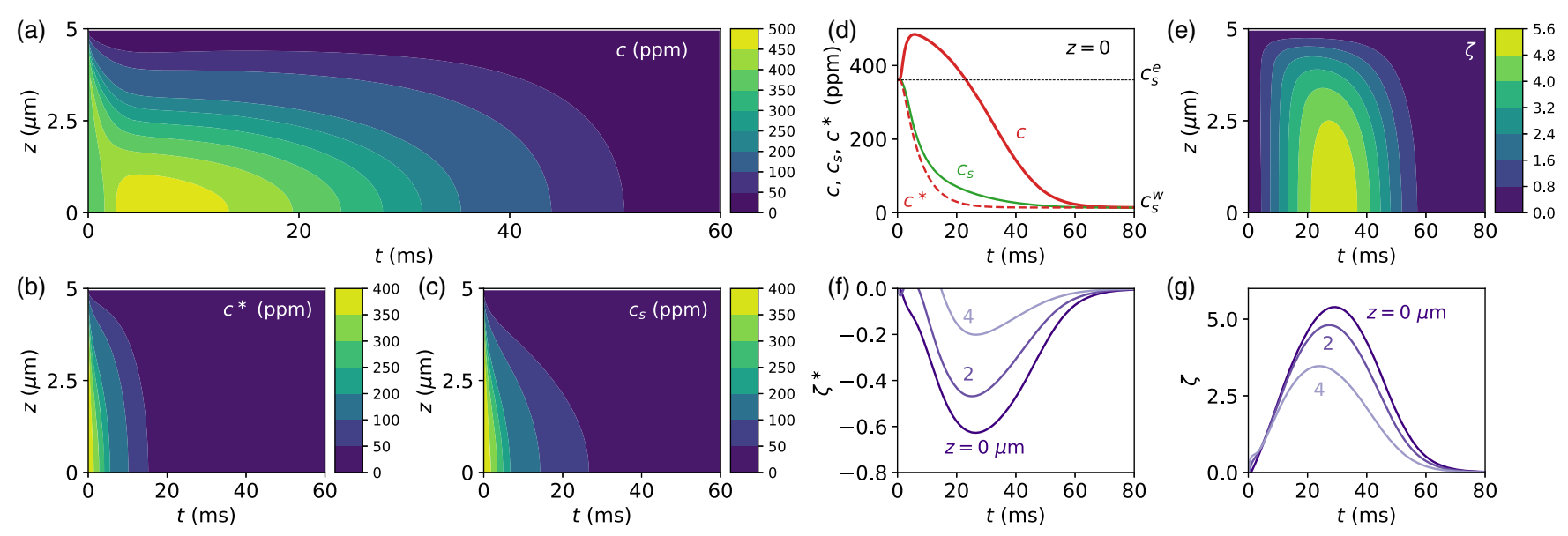

F2:1 FIG. 2. Nitrogen oversaturation is formed during ethanol-water exchange. (a)-(c) Space-time contours of the dissolved nitrogen concentration $c$ in the full model, the concentration $c^{*}$ when counterdiffusion is neglected, and the solubility $c_{s}$. Whereas $c_{s}$ and $c^{*}$ show a monotonous relaxation in time from the saturation concentration of ethanol $c_{s}^{e}$ to that of water $c_{s}^{w}$, oversaturation causes dissolved nitrogen to accumulate at the substrate, before dissipating. (d) The concentration profiles of $c(t), c^{*}(t)$, and $c_{s}(t)$ at the plane of the substrate $z=0$. (e) Space-time contour of oversaturation calculated from Eq. (1). (f)-(g) Temporal evolution of oversaturation pulses in the full model, $\zeta(t)$, and with counterdiffusion neglected, $\zeta^{*}(t)$. The correct directionality (i.e., oversaturation during ethanol-water exchange) is obtained only when counterdiffusion is included in the model.

the two constituent solvents, yielding $c\left(x_{e}\right)=c_{s}^{w}\left(1-x_{e}\right)+$ $c_{s}^{e} x_{e}=c_{s}^{w}+\left(c_{s}^{e}-c_{s}^{w}\right) x_{e}$, which is linearly proportional to $x_{e}$. (The superscripts $w$ and $e$ in $c_{s}$ refer to water and ethanol.) The solubility of the water-ethanol mixture is

$$
c_{s}\left(x_{e}\right) \approx 10^{-5} \times\left(1.39+1.41 x_{e}+33.3 x_{e}^{2}\right),
$$

as empirically determined from experimental measurements [38]; see Fig. 1(b). Comparing both curves pointwise with Eq. (1) yields $\zeta\left(x_{e}\right)$, with maximum $\zeta \approx 1.8$. This formulation has several drawbacks, however. First, it holds only at steady state, ignoring temporal variations in $\zeta$ during mixing. Second, the model is independent of the order of mixing and thus fails to explain the directionality of solvent exchange. We aim to overcome both shortcomings and arrive at a refined estimate through explicit consideration of the transport dynamics of dissolved nitrogen.

To understand the physical picture [Fig. 1(a)], consider initially that a substrate under flow confinement (in experiments typically a microfluidic channel [25] or Hele-Shaw cell $[11,18])$ is initially immersed exclusively in a good solvent of nitrogen, ethanol. Water, a poor solvent of nitrogen, is then flushed into the channel to displace the ethanol. Molecules of ethanol adjacent to the substrate have much smaller velocities than those of the water in the bulk liquid. The mismatch of velocities at the substrate and the bulk liquid establishes a transient concentration boundary layer containing a residual amount of ethanol adjacent to the substrate, which becomes engulfed with water over time [2]. Experiments implicate the involvement of boundary layers during solvent exchange. For example, the volumes of nanodroplets nucleated by the technique $[11,14,18]$ are reproducibly found to scale with Péclet number as
$V \sim \mathrm{Pe}^{3 / 4}$, consistent with the presence of boundary layer with thickness $\ell \propto 1 / \sqrt{\mathrm{Pe}}$ [11].

We now seek to resolve how the concentration field of dissolved nitrogen in the boundary layer evolves as the two solvents mix. We assume that the two solvents and the target species have similar sizes (thus neglecting size effects), that the target species is uncharged, and that it is present only in trace amounts in the liquid. These assumptions are reasonable for the choice of water and ethanol as the solvents, and nitrogen as the dissolved solute, as we consider in this Letter. The large bond dissociation energy of the triple bond in molecular nitrogen prevents spontaneous ionization in water, while nitrogen's solubility is $\sim 10 \mathrm{ppm}$ in water at STP.

As solvent exchange is typically performed within high aspect ratio geometries (e.g., $\sim 1 \mathrm{~cm}$ in lateral extent and $\sim 10 \mu \mathrm{m}$ in confinement height in Ref. [25]) the developed boundary layer can be treated as an approximately flat and semi-infinite domain, whose thickness $\ell$ is determined by the flow conditions. Following scaling estimates from Ref. [11] we set $\ell=5 \mu \mathrm{m}$, but parenthetically note that the essential physics is insensitive to our choice of $\ell$. In a semiinfinite solvent matrix, the concentration $c$ of the nitrogen solute follows the one-dimensional diffusion equation

$$
\frac{\partial c}{\partial t}=D_{n} \frac{\partial^{2} c}{\partial z^{2}} \quad \text { for } z \in[0, \ell],
$$

where $D_{n}$ is the diffusion constant of nitrogen in the liquid matrix. However, Eq. (3) cannot hold if the nitrogen molecule sits in a solvent matrix that is not homogeneous in solubility. To see why, let us briefly outline the derivation of 
Eq. (3). In the liquid phase, the chemical potential of the dissolved nitrogen molecule [30-32,39] is $\mu=$ $\mu_{0}-k_{B} T \ln \left(c / c_{s}\right)$, where $k_{B}$ is the Boltzmann constant and $T$ is the temperature. For a pure solvent, solubility $c_{s}$ is constant, and the force acting on the nitrogen molecule is $F=-\left(k_{B} T / c\right) \nabla c$, which is opposed by a drag force $f v$ ( $f$ is a drag coefficient and $v$ is the particle velocity). Assigning $D_{n} \equiv k_{B} T / f$ and substituting the continuity equation $\partial c / \partial t=-\nabla \cdot(c v)$ leads to Eq. (3). In a heterogeneous binary mixture of solvents, we instead have $F=-\left(k_{B} T / c\right) \nabla c+\left(k_{B} T / c_{s}\right) \nabla c_{s}$ and therefore yield

$$
\frac{\partial c}{\partial t}=D_{n}\left(\frac{\partial^{2} c}{\partial z^{2}}-\frac{c}{c_{s}} \frac{\partial^{2} c_{s}}{\partial z^{2}}\right),
$$

where we define the rightmost term $\left(c / c_{s}\right) \nabla^{2} c_{s}$ as the counterdiffusion term. The solubility field $c_{s}(t, z)$, which we shall specify imminently, is a function of the mole fraction of the two solvents. The physical meaning of Eq. (4) is that the transport of nitrogen solute is governed not only by its own concentration gradient (as it would under diffusion in a homogeneous liquid), but also by the solubility gradient of the liquid solvent matrix. The opposing signs of the diffusion and counterdiffusion terms indicate that the nitrogen and solubility contributions are competitive rather than additive, permitting nitrogen to propagate against its own concentration gradient.

As with the dissolved nitrogen, the mole fraction of ethanol also obeys a one-dimensional diffusion equation

$$
\frac{\partial x_{e}}{\partial t}=D_{e} \frac{\partial^{2} x_{e}}{\partial z^{2}} \quad \text { for } z \in[0, \ell]
$$

where $D_{e}$ is the diffusion constant of ethanol in water, and finally $x_{e}$ is transformed to $c_{s}$ through Eq. (1). Note, however, that whereas the solvent is coupled into the transport equation of nitrogen in Eq. (4), no contribution from the nitrogen solute appears in Eq. (5). This asymmetric effect is a consequence of weak solution theory [3032,39]: the presence of the solvent matrix shifts the chemical potential of the nitrogen by $k_{B} T \ln \left(c / c_{s}\right) \sim$ $0.1-1 k_{B} T$ to the nitrogen solute due to the solvent matrix, but the presence of nitrogen shifts the reciprocal chemical potential of the solvent by a comparatively insignificant $k_{B} T c \sim 10^{-5} k_{B} T \ll k_{B} T$.

For the case of ethanol-water (i.e., good-poor) solvent exchange, the boundary conditions for the coupled equations (4) and (5) are

$$
c(t=0)=c_{s}\left(x_{e}=1\right) \quad \text { and } \quad x_{e}(t=0)=1,
$$

$$
\left.\frac{\partial c}{\partial z}\right|_{z=0}=0 \quad \text { and }\left.\quad \frac{\partial x_{e}}{\partial z}\right|_{z=0}=0,
$$

$$
c(z=\ell)=c_{s}\left(x_{e}=0\right) \quad \text { and } \quad x_{e}(z=\ell)=0 .
$$

In order, these equations state that the boundary layer initially contains ethanol and that the nitrogen concentration in the boundary layer is at the saturation concentration of ethanol; that the substrate is impermeable; and that at the locus of the boundary layer, the nitrogen concentration is at the saturation concentration of water. In the reciprocal case of water-ethanol (i.e., poor-good) solvent exchange, we have

$$
\begin{gathered}
c(t=0)=c_{s}\left(x_{e}=0\right) \quad \text { and } \quad x_{e}(t=0)=0, \\
\left.\frac{\partial c}{\partial z}\right|_{z=0}=0 \quad \text { and }\left.\quad \frac{\partial x_{e}}{\partial z}\right|_{z=0}=0 \\
c(z=\ell)=c_{s}\left(x_{e}=1\right) \quad \text { and } \quad x_{e}(z=\ell)=1 .
\end{gathered}
$$

Solving Eqs. (4) and (5) subject to these boundary conditions yields $c(t, z)$ and $x_{e}(t, z)$, the latter of which is converted to $c_{s}(t, z)$ with Eq. (2). Finally, it should be noted that the diffusion constants $D_{n}$ and $D_{e}$ are themselves functions of the solvent composition, through the mole fraction $x_{e}$. The diffusion constant of the nitrogen in a binary mixture can be reasonably approximated [33] by the geometric mean of the diffusion constant of nitrogen in the two constituent solvents, $D_{n}\left(x_{e}\right)=1 /\left(x_{e} / D_{n}^{e}+\left(1-x_{e}\right) /\right.$ $\left.D_{n}^{w}\right)$. Finally, we obtain the diffusion constant of ethanol in the water-ethanol mixture $D_{e}\left(x_{e}\right)$ by fitting an empirical equation to experimental measurements [34]. The full dependences of $D_{n}\left(x_{e}\right)$ and $D_{e}\left(x_{e}\right)$ are given in Fig. S2 of the Supplemental Material [29].

Good-poor solvent exchange.-To recover the concentration and solubility fields during ethanol-water or goodpoor solvent exchange, we solve Eqs. (4) and (5) subject to boundary conditions [Eqs. (6)-(8)]. In Fig. 2 we show the evolution of $c$ and $c_{s}$ in both space $z$ and time $t$ in contour plots. Both quantities start at the saturation concentration of nitrogen in ethanol $c_{s}^{e}$, and equilibrate toward the saturation concentration of nitrogen in water, $c_{s}^{w}$ [see Fig. 2(d)].

There is, however, a noticeable difference in how $c$ and $c_{s}$ relax toward equilibrium, leading to the creation of substantial oversaturation. Whereas the relaxation of $c_{s}$ is purely diffusive and therefore monotonous, the corresponding relaxation of $c$ is not. Of particular relevance to nucleation processes is the evolution of nitrogen concentration at the plane of the substrate, $z=0$. We observe that $c$ increases beyond $c_{s}^{e}$, reaches a maximum, and only then finally relaxes to $c_{s}^{w}$. Applying Eq. (1), we are able to resolve also the development of oversaturation in space and time. From the contour plot [Fig. 2(e)] of $\zeta$ we see that the oversaturation is considerable in magnitude, reaching a maximum of $\zeta \approx 5.6$, substantially larger than the $\zeta \approx 1.8$ predicted by the An model [27]. The oversaturation also persists over a large spatial extent, as $\zeta>4.8$ for about half of the boundary layer, before decaying to zero due to the boundary condition at the boundary layer [Eq. (8)]. 

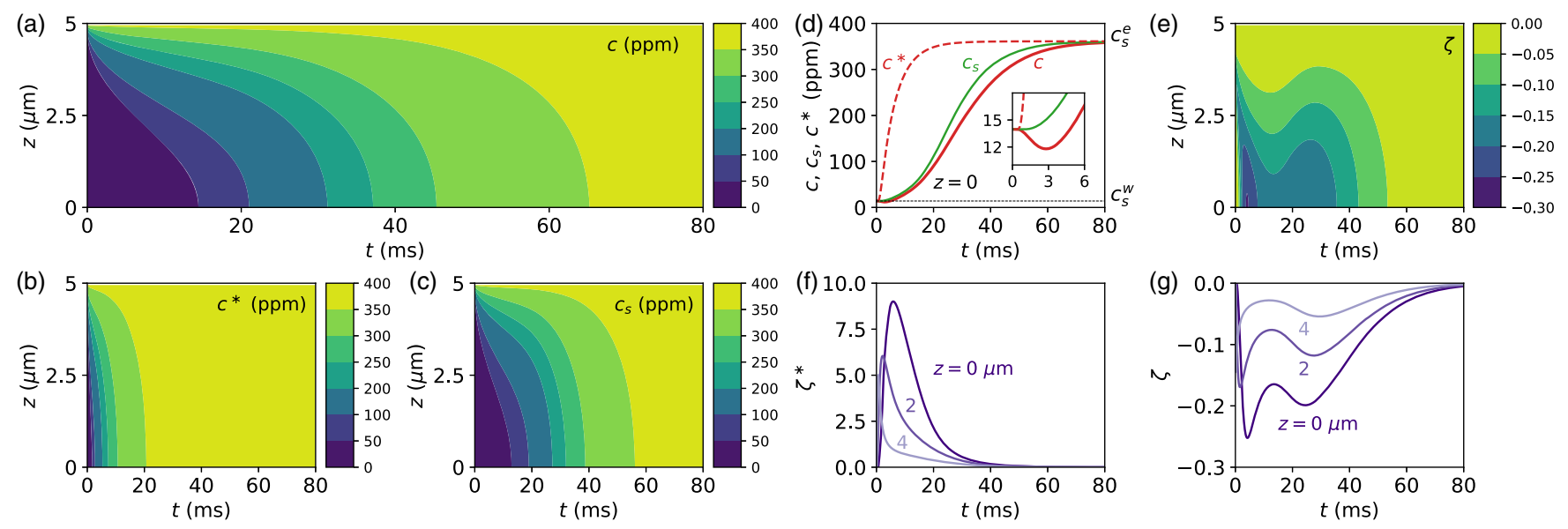

FIG. 3. Water-ethanol exchange prompts undersaturation of dissolved nitrogen. (a)-(c) Space-time contours of the dissolved nitrogen concentration $c$ in the full model, the nitrogen concentration $c^{*}$ when counterdiffusion is neglected, and the solubility $c_{s}$ of the solvent mixture. (d) Temporal evolution of $c$ (solid line) and $c_{s}$ (dashed line) at the wall, $z=0$. The inset shows that $c^{*}$ and $c_{s}$ both relax from $c_{s}^{w}$ to $c_{s}^{e}$ monotonously, but $c$ dips slightly from counterdiffusion before relaxation. This is the counterpart to the strong nonmonotonous increase in $c$ in ethanol-water exchange in Fig. 2(d). (e) Space-time contours of oversaturation generated in the full model, applying Eq. (1) to (a) and (c). (f),(g) Oversaturations $\zeta$ and $\zeta^{*}$ produced in the full model and with counterdiffusion neglected respectively at varying separations from the substrate. As with the ethanol-water case, the experimentally observed directionality is observed only when counterdiffusion is incorporated.

Poor-good solvent exchange.-Next, we consider the reciprocal case of water-ethanol or poor-good solvent exchange, again solving Eqs. (4) and (5) but subject to boundary conditions Eqs. (9)-(11). Here, as seen in Figs. 3(a) and 3(c), respectively, both $c$ and $c_{s}$ relax from $c_{s}^{w}$ to $c_{s}^{e}$. A profile of the oversaturation at the substrate $\zeta(t, z=0)$ [see Fig. 3(d)] shows that $c$ and $c_{s}$ track each other relatively closely. Since $c<c_{s}$, calculating the oversaturation contours $\zeta$ from Eq. (1) [Fig. 3(e)] reveals that water-ethanol exchange produces undersaturation in the boundary layer, with the strongest undersaturation of $\zeta \approx$ -0.25 produced at the plane of the substrate [Fig. 3(f)].

Counterdiffusion.-Our calculations so far show that ethanol-water exchange produces substantial oversaturation, but the reciprocal exchange undersaturates the liquid. To understand how counterdiffusion influences these outcomes, we solve Eqs. (4) and (5) with counterdiffusion omitted and denoting the uncoupled nitrogen concentration field as $c^{*}$. The space-time contours of $c^{*}$ are shown in Figs. 2(b) and 3(b), and profiles of $c^{*}(t)$ at $z=0$ are shown in Figs. 2(d) and 3(d).

In the absence of counterdiffusion, the aforementioned outcomes of solvent exchange are exactly inverted. Neglecting counterdiffusion amounts to uncoupling $c^{*}$ and $c_{s}$, with the outcome of solvent exchange (i.e., whether $\zeta<0$ or $\zeta>0$ ) depending only on the competing diffusive timescales of the two problems. For water-ethanol-nitrogen, $D_{e}<D$ for all $x_{e}$ (Fig. S2 of the Supplemental Material [29]) and $c^{*}$ will reach steady-state more quickly than $c_{s}$ does in both directions, creating oversaturation $\left(c^{*}>c_{s}\right)$ during water-ethanol exchange and undersaturation $\left(c^{*}<c_{s}\right)$ during ethanol-water exchange. The physical picture of the dynamics in the absence of counterdiffusion is that when a substrate wetted by ethanol is replaced by water, the concentration gradient-along which nitrogen is transported-directs away from the substrate; reciprocal arguments apply for the reverse exchange.

Substrate.-The substrate plays an important but previously neglected role in solvent exchange. During ethanolwater exchange, nitrogen molecules are advected toward the confining boundary at $z=0$, but cannot leave the system there, leading to a focusing effect that accumulates nitrogen adjacent to the substrate. Accumulation is only transient because nitrogen molecules continuously leave the computational domain through the other boundary at $z=\ell$. In contrast, during water-ethanol exchange the nitrogen is advected away from the substrate and toward the $z=\ell$ boundary, and the confining effect is absent here. This explains the quantitative differences between the strong ethanol-water oversaturation in Fig. 2(d) and the modest water-ethanol undersaturation in Fig. 3(d) $-c(t)$ and $c_{s}(t)$ track each other closely in the former but exhibit large quantitative differences in the latter.

Our model compares well with both experiments and molecular dynamics (MD) simulations. Apart from recovering the experimentally observed directionality of solvent exchange repeatably reported by many groups, our model predicts a pulselike variation of oversaturation during ethanol-water exchange. So-called "oversaturation pulses" at mixing fronts have been observed both experimentally with brightfield microscopy [11] and in intricate MD simulations [40] of the good-poor solvent exchange. A direct quantitative comparison with MD is not possible due to the unavailability of $D_{n}\left(x_{e}\right)$ and $D_{e}\left(x_{e}\right)$ as well as the 
use of unrealistically high $\zeta$ in simulations [41], violating the weak solution assumption we use. Nevertheless, our model recovers qualitatively similar oversaturation profiles to the simulations in Fig. 2 of Ref. [40], and quantitatively accounts for their observation that in good-poor exchange, dissolved nitrogen advects toward the substrate, against its own concentration gradient.

In summary, we present a minimal model for the solvent exchange of water and ethanol. We argue that transient solubility gradients are responsible for strong accumulation of dissolved nitrogen during ethanol-water exchange, and conversely, evacuation during water-ethanol exchange. Solvent exchange is a particularly simple and efficacious technique to produce nanobubbles and nanodroplets at scale, but as a nucleation-driven process, quantitative control of this process is an ongoing challenge. Our results elucidate the salient physical mechanisms of solvent exchange and offer a means to quantitatively predict oversaturation in this system, therefore paving the way for achieving size selectivity of surface nanobubbles and nanodroplets in a variety of chemical engineering processes such as purification, separation, or remediation of multicomponent mixtures.

B. H. T. thanks Duoming Chen and Xianyue Chen for sharing key insights. C. D. O. is supported by a joint research program between the Natural Science Foundation of China (Program No. 11861131005) and the Deutsche Forschungsgemeinschaft of Germany (Program No. OH 75/3-1). H. A. acknowledges the support of a Future Fellowship from the Australian Research Council.

*claus-dieter.ohl@ovgu.de

†hongjie.an@griffith.edu.au

[1] D. Lohse and X. Zhang, Surface nanobubbles and nanodroplets, Rev. Mod. Phys. 87, 981 (2015).

[2] D. Lohse and X. Zhang, Physicochemical hydrodynamics of droplets out of equilibrium, Nat. Rev. Phys. 2, 426 (2020).

[3] W. Feng, E. Ueda, and P. A. Levkin, Droplet microarrays: From surface patterning to high-throughput applications, Adv. Mater. 30, 1706111 (2018).

[4] E. Karatay, A. S. Haase, C. W. Visser, C. Sun, D. Lohse, P. A. Tsai, and R. G. Lammertink, Control of slippage with tunable bubble mattresses, Proc. Natl. Acad. Sci. U.S.A. 110, 8422 (2013).

[5] P.-G. de Gennes, On fluid/wall slippage, Langmuir 18, 3413 (2002).

[6] T. Ondarçuhu and J.-P. Aimé, Nanoscale Liquid Interfaces: Wetting, Patterning and Force Microscopy at the Molecular Scale (Jenny Stanford Publishing, Singapore, 2013)—an excellent account of the state of-the-art in nanodroplet fabrication is given in Chapter 12 .

[7] A. Checco, P. Guenoun, and J. Daillant, Nonlinear Dependence of the Contact Angle of Nanodroplets on Contact Line Curvature, Phys. Rev. Lett. 91, 186101 (2003).

[8] J.-U. Park, J.H. Lee, U. Paik, Y. Lu, and J. A. Rogers, Nanoscale patterns of oligonucleotides formed by electrohydrodynamic jet printing with applications in biosensing and nanomaterials assembly, Nano Lett. 8, 4210 (2008).

[9] A. Fang, E. Dujardin, and T. Ondarçuhu, Control of droplet size in liquid nanodispensing, Nano Lett. 6, 2368 (2006).

[10] X.H. Zhang and W. Ducker, Interfacial oil droplets, Langmuir 24, 110 (2008).

[11] X. Zhang, Z. Lu, H. Tan, L. Bao, Y. He, C. Sun, and D. Lohse, Formation of surface nanodroplets under controlled flow conditions, Proc. Natl. Acad. Sci. U.S.A. 112, 9253 (2015).

[12] Q. Zhao, D. W. Lee, B. K. Ahn, S. Seo, Y. Kaufman, J. N. Israelachvili, and J. H. Waite, Underwater contact adhesion and microarchitecture in polyelectrolyte complexes actuated by solvent exchange, Nat. Mater. 15, 407 (2016).

[13] A. Nikoubashman, V. E. Lee, C. Sosa, R. K. Prudhomme, R. D. Priestley, and A.Z. Panagiotopoulos, Directed assembly of soft colloids through rapid solvent exchange, ACS Nano 10, 1425 (2016).

[14] C. Xu, H. Yu, S. Peng, Z. Lu, L. Lei, D. Lohse, and X. Zhang, Collective interactions in the nucleation and growth of surface droplets, Soft Matter 13, 937 (2017).

[15] B. Dyett, A. Kiyama, M. Rump, Y. Tagawa, D. Lohse, and X. Zhang, Growth dynamics of surface nanodroplets during solvent exchange at varying flow rates, Soft Matter 14, 5197 (2018).

[16] B. Dyett, H. Hao, D. Lohse, and X. Zhang, Coalescence driven self-organization of growing nanodroplets around a microcap, Soft Matter 14, 2628 (2018).

[17] H. Yu, M. Rump, S. Maheshwari, L. Bao, and X. Zhang, Growth of nanodroplets on a still microfiber under flow conditions, Phys. Chem. Chem. Phys. 20, 18252 (2018).

[18] B. Zeng, Y. Wang, X. Zhang, and D. Lohse, Solvent exchange in a hele-shaw cell: Universality of surface nanodroplet nucleation, J. Phys. Chem. C 123, 5571 (2019).

[19] Q. Xie and J. Harting, The effect of the liquid layer thickness on the dissolution of immersed surface droplets, Soft Matter 15, 6461 (2019).

[20] S.-T. Lou, Z.-Q. Ouyang, Y. Zhang, X.-J. Li, J. Hu, M.-Q. Li, and F.-J. Yang, Nanobubbles on solid surface imaged by atomic force microscopy, J. Vac. Sci. Technol., B: Microelectron. Nanometer Struct.-Process., Meas., Phenom. 18, 2573 (2000).

[21] A. C. Simonsen, P. L. Hansen, and B. Klösgen, Nanobubbles give evidence of incomplete wetting at a hydrophobic interface, J. Colloid Interface Sci. 273, 291 (2004).

[22] X. Zhang, Z. Wu, X. Zhang, G. Li, and J. Hu, Nanobubbles at the interface of HOPG and ethanol solution, Int. J. Nanosci. 4, 399 (2005).

[23] S. Yang, S. M. Dammer, N. Bremond, H. J. Zandvliet, E. S. Kooij, and D. Lohse, Characterization of nanobubbles on hydrophobic surfaces in water, Langmuir 23, 7072 (2007).

[24] S. Karpitschka, E. Dietrich, J. R. T. Seddon, H. J. W. Zandvliet, D. Lohse, and H. Riegler, Nonintrusive Optical Visualization of Surface Nanobubbles, Phys. Rev. Lett. 109, 066102 (2012).

[25] C. U. Chan and C.-D. Ohl, Total-Internal-ReflectionFluorescence Microscopy for the Study of Nanobubble Dynamics, Phys. Rev. Lett. 109, 174501 (2012). 
[26] N. Hain, D. Wesner, S. I. Druzhinin, and H. Schonherr, Surface nanobubbles studied by time-resolved fluorescence microscopy methods combined with afm: The impact of surface treatment on nanobubble nucleation, Langmuir 32, 11155 (2016).

[27] H. An, G. Liu, R. Atkin, and V. S. J. Craig, Surface nanobubbles in nonaqueous media: Looking for nanobubbles in dmso, formamide, propylene carbonate, ethylammonium nitrate, and propylammonium nitrate, ACS Nano 9, 7596 (2015).

[28] L. Zargarzadeh and J. A. Elliott, Thermodynamics of surface nanobubbles, Langmuir 32, 11309 (2016).

[29] See Supplemental Material at http://link.aps.org/ supplemental/10.1103/PhysRevLett.000.000000 for additional calculations, information about diffusion constants, and a discussion on convection effects, which includes Refs. [1,11,28,30-37].

[30] C. Ward, A. Balakrishnan, and F. Hooper, On the thermodynamics of nucleation in weak gas-liquid solutions, J. Basic Eng. 92, 695 (1970).

[31] C. Ward and A. Tucker, Thermodynamic theory of diffusion-controlled bubble growth or dissolution and experimental examination of the predictions, J. Appl. Phys. 46, 233 (1975).

[32] C. Ward and E. Levart, Conditions for stability of bubble nuclei in solid surfaces contacting a liquid-gas solution, J. Appl. Phys. 56, 491 (1984).

[33] R. B. Bird, W. E. Stewart, and E. N. Lightfoot, Transport Phenomena (John Wiley \& Sons, New York, 1960).
[34] K. Pratt and W. Wakeham, The mutual diffusion coefficient of ethanol-water mixtures: Determination by a rapid, new method, Proc. R. Soc. A 336, 393 (1974).

[35] J. R. Seddon and D. Lohse, Nanobubbles and micropancakes: Gaseous domains on immersed substrates, J. Phys. Condens. Matter 23, 133001 (2011).

[36] R. Colaço, A. Serro, and B. Saramago, On the stability of bubbles trapped at a solid-liquid interface: A thermodynamical approach, Surf. Sci. 603, 2870 (2009).

[37] J. R. T. Seddon and H. J. W. Zandvliet, Comment on: on the stability of bubbles trapped at a solid-liquid interface: A thermodynamical approach, Surf. Sci. 604, 476 (2010).

[38] R. Battino, T. R. Rettich, and T. Tominaga, The solubility of nitrogen and air in liquids, J. Phys. Chem. Ref. Data 13, 563 (1984).

[39] L. D. Landau and E. Lifshitz, Statistical Physics, part 1: Volume 5 (Butterworth-Heinemann, Oxford, 1980).

[40] Q. Xiao, Y. Liu, Z. Guo, Z. Liu, D. Lohse, and X. Zhang, Solvent exchange leading to nanobubble nucleation: A molecular dynamics study, Langmuir 33, 8090 (2017).

[41] The mole fraction of water-dissolved nitrogen is $f \sim 10^{-5}$, requiring exorbitant system sizes. In practice [42], MD simulations use $f \sim 10^{-2}$ (equivalently $\zeta \sim 10^{3}$ ) instead.

[42] L. Wang, X. Wang, L. Wang, J. Hu, C. L. Wang, B. Zhao, X. Zhang, R. Tai, M. He, and L. Chen, Formation of surface nanobubbles on nanostructured substrates, Nanoscale 9 , 1078 (2017). 